\title{
VARIATION OF BROAD OPTICAL EMISSION LINES IN AGN'S.
}

\author{
N.G. BOCHKAREV \\ Sternberg Astronomical Institute, Moscow 119899, Russia \\ A.I. SHAPOVALOVA \\ Special Astrophysical Observatory, N.Arkhyz, Stavropolskii Krai, 357147, Russia \\ and \\ L.S.NAZAROVA \\ Royal Greenwich Observatory, Cambridge, CB30EZ,UK
}

\begin{abstract}
.
AGN optical spectra $(4000-5000 \AA)$ have been obtained with the TV scanner of the 6-m telescope at Special Astrophysical Observatory (N.Arkhiz-North Caucasus) from 1986 to now. Most spectra were obtained with dispersion of $1 \AA /$ channel and with $3-4 \AA$ spectral resolution and signal-to-noise ratio $\mathrm{S} / \mathrm{N}=10-25$ for individual spectra. NGC 4151 , NGC 3516, NGC 5548 and NGC 7469 are the main sources of the programme and are also being used in international AGN watch programmes for diagnosis, estimation of the size and structure of the BLR. NGC4151:

In 1987 the broad components of the $H \beta$ and $H \gamma$ lines decreased strongly (on about $65 \%$ as compared with 1986) and fell to a level comparable to that of 1984 . In 1988 they increased up approximately to the level of 1986 . During the photometrically low state of NGC 4151 satellites of the strong $H \beta, H \gamma, H \delta$ and He II $4686 \AA$ lines were suspected at radial velocities of $+9400 \mathrm{~km} / \mathrm{s}$ and $-7500 \mathrm{~km} / \mathrm{s}$. The data are could be interprite a two-jet model with jet velocity of 8400 $24000 \mathrm{~km} / \mathrm{s}$. (Bochkarev et al.,1989). Analysis of the spectra shows that the broad component of HeII $\lambda 4686 \AA$ changed its asymmetry over 6 years. Bochkarev et al.(1992), Nazarova et al.(1993) discussed a possible explanation of profile HeII $\lambda 4686 \AA$ behavior by involving a precession of cones of ionizing radiation.

NGC5548:

Cross-correction of the optical emission line light curves with the UV continuum light curve reveals that the lines respond to continuum variations with a time delay from 7 days for HeII $4686 \AA$ to 18 days for $H \beta$. $H \gamma$ responds more rapidly than the lower order Balmer lines and there is a general trend towards higher amplitude variations in higher-order lines. The time lags for the line variations are consistent with the general pattern of radial ionization stratification of the BLR. During 1988-89 Fe II in blend 4130 varies with an amplitude of aproximately $20 \%$ about the mean, similar the amplitude of the $H \beta$ variations and Balmer continuum.

NGC3516:

Variations of $H \beta$ during 1986-88 were small (20\%), the shape of the profile (including the assymetry) did not change, but $H \gamma$ - to $H \beta$ ratios of the profile integrated intensities of broad component increased from 0.43 in 1986 to 0.8 in 1988 . The $30 \%$ variations of continuum on characteristic time scale 10-15 days during the spring of 1988 were not accompanied by any noticeable changes in the line profile (Bochkarev et al.,1990).
\end{abstract}

Key words: data analysis-galaxies:NGC4151, NGC5548, NGC3515-galaxies: Seyfert-lines:profilespectroscopy

\section{References}

N.G.Bochkarev, A.I.Shapovalova, S.A.Zhekov,1989,Preprint Special Astrophysical obs.,N35

N.G.Bochkarev, A.I.Shapovalova, S.A.Zhekov,1990,Preprint Special Astrophysical obs.,N45

N.G.Bochkarev, L.S.Nazarova, A.I.Shapovalova, 1992,Astron. and Astroiphys.Trans.,v.1, No.3.

L.S.Nazarova, N.G.Bochkarev, A.I.Shapovalova,(in press)

T. J.-L. Courvoisier and A. Blecha: Multi-Wavelength Continuum Emission of AGN, 439.

(C) 1994 IAU. Printed in the Netherlands. 